\title{
Freeze out, IR-catastrophes and Non-thermal Emission in SNe
}

\author{
By CLAES FRANSSON, JOHN HOUCK, \\ AND CECILIA KOZMA
}

\author{
Stockholm Observatory, S-133 36 Saltsjöbaden, Sweden
}

\begin{abstract}
Freeze out effects and the IR-catastrophe are discussed for SN 1987A and for Type Ia SNe. We show that the light curves of the optical lines in SN 1987A provide strong evidence for the IR-catastrophe. We also argue that most optical lines are dominated by non-thermal excitation after $\sim 800$ days. The level of this emission is set mainly by the total mass of the elements. Models of the [O I] $\lambda \lambda 6300-64$ light curve show that an oxygen mass of $\sim 1.5 \mathrm{M}_{\odot}$ is needed. Light curve models for Type Ia SNe display a sharp decrease in the optical flux as a result of the IR-catastrophe at $\sim 500$ days, producing UBV-photometry inconsistent with observations of SN 1972E by Kirshner \& Oke (1975).
\end{abstract}

\section{Introduction}

Observations of SN 1987A, but also a number of other Type II and Type Ia SNe, at late stages have made it possible to study a number of new features in the evolution of the SN ejecta from explosion to the remnant stage. Here we discuss some recent results in this evolution. A more complete review of the background physics can be found in Fransson (1993).

\section{SN 1987A}

It is now well established from the bolometric light curve that $\sim 0.07 \mathrm{M}_{\odot}$ of ${ }^{56} \mathrm{Ni}$ was created in SN 1987A, and that this is responsible for most of the observed emission from the SN during the first $\sim 800$ days. Being based on the bolometric light curve, this is a fairly model independent conclusion. However, to extract any detailed information about abundances or the physical conditions from the spectrum, an understanding of the thermalization process of the $\gamma$-rays is necessary. The first step in this process, in which the photons are degraded by Compton scattering, creating a number of non-thermal primary keV-electrons, is fairly straight forward, and can be calculated by e.g. Monte Carlo methods (e.g. Pinto \& Woosley 1988). The subsequent slow-down of the electrons and the cascade of secondary electrons is considerably more complicated. Kozma \& Fransson (1992, in the following KF92) and Xu \& McCray (1991) have modeled this, using the Spencer-Fano formalism. The absorbed $\gamma$-ray energy is divided into three channels: $H_{\gamma}$, heating the thermal electrons; $\sum_{i} \Phi_{i}$, producing ionizations of the ions $i$; and $\sum_{i, j} \epsilon_{j, i}$, producing excitations of levels $j$ in the ions $i$. At low electron fractions these three channels are roughly equal, but above $x_{e} \sim 0.1$ ionizations and excitations decrease rapidly, and most of the energy goes into heating. To relate the number of ionizations per ion to $\Phi_{i}$ it is convenient to define an effective ionization potential,

$$
\chi_{e f f, i}=\frac{\chi_{i}}{\Phi_{i} X_{i}}
$$

where $X_{i}$ is the relative abundance of the ion and $\chi_{i}$ the ionization potential.

As the ejecta expand and the $\gamma$-input decreases, the number density, $n_{i}$ of an ion $i$ is 
determined by

$$
\begin{aligned}
\frac{d n_{i}}{d t}+\frac{3 n_{i}}{t}= & \left\{4 \pi \frac{J_{\gamma} \sigma_{\gamma, i}}{\chi_{e f f, i}}+4 \pi \int_{\nu_{0}}^{\infty} \frac{J_{\nu} \sigma_{\nu, i}}{h \nu} d \nu+\sum_{k} \zeta_{k+1, i} n_{k+1}\right\} n_{i} \\
& +\left\{\alpha_{i+1} n_{e}+\sum_{k} \zeta_{k, i+1} n_{k}\right\} n_{i+1}
\end{aligned}
$$

The term on the left represents the change due to expansion. The first term on the right is the $\gamma$-ray ionization rate, proportional to the mean intensity of the $\gamma$-rays, $J_{\gamma}$, and the cross-section for $\gamma$-ray scattering, $\sigma_{\gamma, i}=\kappa_{\gamma} A_{i} m_{p}$ with the mass absorption coefficient $\kappa_{\gamma}=0.06 Z_{i} / A_{i} \mathrm{~cm}^{2} \mathrm{~g}^{-1}\left(A_{i}\right.$ and $Z_{i}$ are the mass and atomic number of the element). The second term is the rate of photoionization by diffuse emission from line and continuum photons in the remnant. The third term represents ionizations due to charge transfer between the ions $i$ and $k+1$, with rate $\zeta_{k+1, i}$. The next terms are the inverse processes due to recombination and charge transfer involving the higher ionization stage $i+1$. Most of the required effective ionization potentials, $\chi_{e f f, i}\left(x_{e}\right)$, can be found in KF92. The charge transfer rates for reactions involving hydrogen and helium are fairly well known. However, for reactions between different metals they are to a large extent unknown, and one has to guess rates based on the positions of the energy levels in the ions involved. This is a major uncertainty in the modeling. If a near-resonance situation is present the rate coefficient is likely to be $\sim 10^{-10} \mathrm{~cm}^{3} \mathrm{~s}^{-1}$, or higher. The total rate, $n_{i+1} \zeta_{k, i+1}$, may therefore easily dominate over radiative recombinations, which have rate coefficients proportional to $x_{e} \alpha_{r e c} \approx 10^{-2} \times 10^{-12}=10^{-14} \mathrm{~cm}^{3} \mathrm{~s}^{-1}$.

Neglecting photoionization and charge transfer, and assuming that the atomic time scales are shorter than the expansion time scale and the radioactive decay time scale, we can solve equation (2.2) for the electron fraction, using $n_{e} \approx n_{i+1}$,

$$
x_{e} \approx\left(\frac{4 \pi J_{\gamma} \sigma_{\gamma, i}}{\chi_{e f f, i} \alpha_{i+1} n_{t o t a l}}\right)^{1 / 2},
$$

assuming that $x_{e}<<1$. The $\gamma$-ray mean intensity is given by

$$
J_{\gamma}=\frac{D_{\gamma} L_{\gamma}}{16 \pi^{2} r^{2}}=\frac{D_{\gamma} L_{\gamma}}{16 \pi^{2}(V t)^{2}},
$$

where $D_{\gamma}$ is a geometric factor accounting for the distribution of the radioactive energy source (KF92), and

$$
L_{\gamma}=C e^{-t / \tau}=1.3 \times 10^{42}\left(\frac{M\left({ }^{56} \mathrm{Ni}\right)}{0.1 \mathrm{M}_{\odot}}\right) e^{-t / 111.3} \mathrm{ergs}
$$

if ${ }^{56} \mathrm{Co}$ dominates the energy input. Assuming that the zone has a filling factor $f_{i}$ and mass $M_{i}$, we find

$$
\begin{aligned}
x_{e} \approx & C_{i} f_{i}^{1 / 2} D_{\gamma}^{1 / 2}\left(\frac{M\left({ }^{56} \mathrm{Ni}\right)}{0.1 \mathrm{M}_{\odot}}\right)^{1 / 2}\left(\frac{M_{i}}{\mathrm{M}_{\odot}}\right)^{-1 / 2} \times \\
& \left(\frac{V_{i}}{2000 \mathrm{~km} \mathrm{~s}^{-1}}\right)^{1 / 2}\left(\frac{t}{1000 \mathrm{~d}}\right)^{1 / 2} e^{-t / 222 \mathrm{~d}}
\end{aligned}
$$

where $C_{H}=0.15$ for $\mathrm{H}, C_{H e}=0.24$ for $\mathrm{He}$, and $C_{O}=1.28$ for $\mathrm{O}$. For Type II cores we therefore find that $x_{e}<<1$. The recombination time, $t_{r e c}=1 /\left(n_{e} \alpha_{r e c}\right)$, increases as $t_{r e c} \propto t^{5 / 2} e^{t / 2 \tau}$. Eventually, this time scale must become longer than the radioactive decay time scale, $\tau$, or even the expansion time scale, $t$. This defines the freeze out time (Fransson \& Kozma 1993, FK93). The recombination time scale varies quite strongly 
from region to region in the core. In the metal rich regions $x_{e}$ is higher than in the $\mathbf{H}$ and $\mathrm{He}$ zones, mainly because these regions have fewer atoms per unit mass. Freeze-out effects are therefore strongest in the hydrogen and helium rich regions in the core, and especially in the envelope. For parameters typical of SN 1987A freeze out occurs after $800-900$ days in these zones. If the density in the ejecta is highly inhomogeneous, as is indicated from mixing calculations, freeze out effects may dominate the evolution in the low density regions, because $t_{r e c} \propto n_{\text {total }}^{-1 / 2}$. The effects of the freeze out for the light curve are discussed in FK93. Here we are mainly concerned with the temperature evolution and the emission from the ejecta.

After $~ 800$ days radioactive input from ${ }^{57}$ Co becomes an important energy source if the ${ }^{57} \mathrm{Ni} /{ }^{56} \mathrm{Ni}$ ratio is $1.5-2$ times solar, as is indicated by $\gamma$-ray observations (Kurfess et al. 1992). After this epoch the recombination and cooling time scales should be compared with the ${ }^{57}$ Co decay time $\tau=391$ days. Calculations of the nucleosynthesis indicate that $\sim 10^{-4} \mathrm{M}_{\odot}$ of ${ }^{44} \mathrm{Ti}$ is also produced in the explosion, which should dominate after $\sim 1500$ days. Most of this energy is emitted as positrons, which are likely to be stopped on the spot in the Fe rich gas, resulting in Fe I and Fe II emission. Most of this will be in the far-IR. Even if the $\gamma$-ray input is small in the other regions there will still be emission from the Co-poor regions due to the freeze out.

The temperature is determined by

$$
\frac{d T_{e}}{d t}=\frac{2}{3 k\left(1+x_{e}\right)}\left(4 \pi H_{\gamma} J_{\gamma} \sigma_{\gamma, i}-n_{e} \Gamma\left(T_{e}\right)\right)-\frac{2 T_{e}}{t}-\frac{2 T_{e}}{\left(1+x_{e}\right)} \frac{d x_{e}}{d t}
$$

where $\Gamma\left(T_{e}\right)$ is the total cooling rate. The $2 T_{e} / t$ term is due to adiabatic expansion, and shows that $T_{e} \propto t^{-2}$, if the cooling and heating time scales are longer than $t . \Gamma\left(T_{e}\right)$ is the sum of a large number of contributions involving collisional excitation by the thermal electrons in the gas. At temperatures above $\sim 2000 \mathrm{~K}$ excitations of forbidden, optical and near-IR lines dominate the cooling. Some important examples are [O I] $\lambda \lambda 6300-64$, $[\mathrm{Ca} \mathrm{II}] \lambda 7300$, and $[\mathrm{Si} \mathrm{I}] \lambda \lambda 1.099,1.645 \mu$. H $\alpha$ does not contribute much to the cooling and arises mainly as a result of recombination (see below). Below $\sim 2000 \mathrm{~K}$ the temperature is too low to excite the optical lines, and far-IR fine structure transitions dominate. Some of the strongest are [Ne II] $\lambda 12.81 \mu,[\mathrm{Si} \mathrm{II}] \lambda 34.81 \mu,[\mathrm{Fe} \mathrm{I}] \lambda \lambda 24.05,34.72 \mu$, $[\mathrm{Fe} \mathrm{II}] \lambda \lambda 25.99,35.35 \mu$ and $[\mathrm{O} \mathrm{I}] \lambda 63.00 \mu$.

The temperature and ionization histories depend strongly on the chemical composition. Microscopic mixing is probably not very efficient (Fryxell, Müller \& Arnett 1991), therefore both $T_{e}$ and $x_{e}$ are different for each chemical composition zone (Fransson $\&$ Chevalier 1989). We have calculated the temperature and ionization structure for a simplified model consisting of an inhomogeneous core with five different chemical zones expanding with a velocity of $1500 \mathrm{~km} \mathrm{~s}^{-1}$, plus a hydrogen envelope. Each of the core zones have a total mass $M_{i}$ and a filling factor $f_{i}$, while the envelope has a density profile taken from the Shigeyama, Nomoto \& Hashimoto (1988) 14E1 model. The core consists of $0.07 \mathrm{M}_{\odot}$ of ${ }^{56} \mathrm{Ni}$, decaying into $\mathrm{Co}$ and $\mathrm{Fe}$, with $f_{N i}=0.29,0.06 \mathrm{M}_{\odot}$ from the $\mathrm{Si}-\mathrm{Ca}$ zone, with $f_{S i}=0.01,2.0 \mathrm{M}_{\odot}$ from the $\mathrm{O}$ zone, with $f_{O}=0.30,2.0 \mathrm{M}_{\odot}$ from the He zone, with $f_{H e}=0.20$, and $2 \mathrm{M}_{\odot}$ from mixed H-rich gas, with $f_{H}=0.20$. The mass of the $\mathrm{H}$ envelope is $10 \mathrm{M}_{\odot}$. Although crude, this mimics the expected macroscopic mixing of the core.

In figure 1 we show the temperature evolution of each of these core zones, and in figure 2 the envelope temperature structure for a few dates. The evolution of the metal rich zones are fairly similar, with a smooth decrease in temperature until $\sim 900$ days, when a drastic decrease in temperature occurs. This is often referred to as the IR-catastrophe (Axelrod 


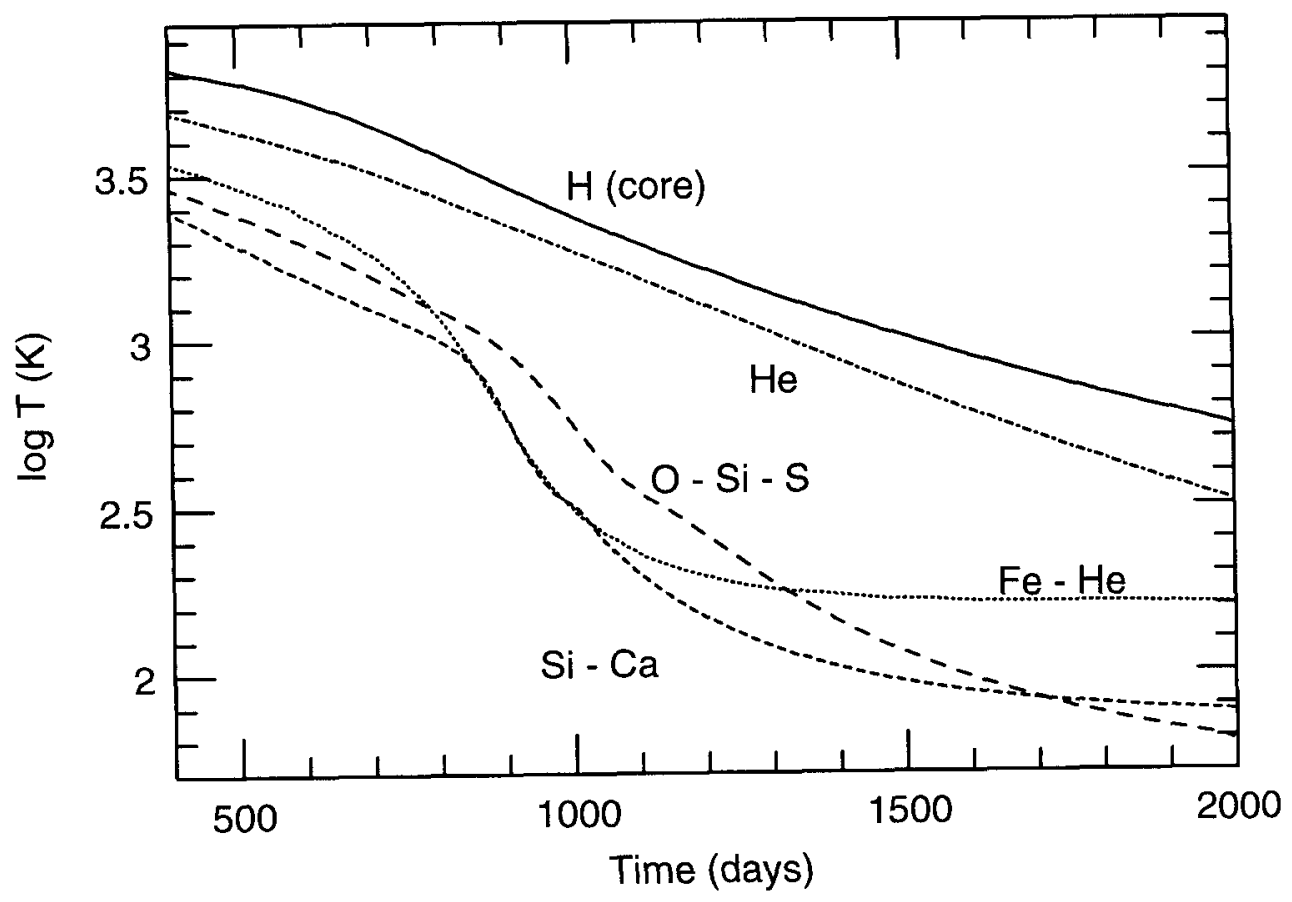

Figure 1. Temperature in the different zones as a function of time. In the metal rich zones a thermal instability is obvious at $\sim 900$ days, while the hydrogen and helium rich zones cool adiabatically

1980; Fransson \& Chevalier 1989). A qualitative discussion of the basic physics can be found in Fransson (1993). In the $\mathrm{H}$ - and He-rich gas the temperature decreases more smoothly, and has an evolution characteristic of an adiabatic gas, $T_{e} \propto t^{-2}$. The same is also true for the envelope, where the temperature decreases outward. The temperatures in the hydrogen gas agree well with those reported by Chugai \& Danziger (1993) at this conference, based on the width of the Paschen continuum.

Although the IR-catastrophe has long been predicted, observational evidence has been rather scarce. However, clear evidence can be seen in observations of the emission line fluxes at late times. The emitted radiation from an ion $i$ and from a level $j$ can be split schematically into three contributions

$$
L_{i j} \propto h \nu_{i j} C_{i j}\left(T_{e}\right) n_{e} n_{i}+j_{r e c, i j} n_{e} n_{i+1}+4 \pi \epsilon_{i j} J_{\gamma} \sigma_{\gamma, i} n_{i},
$$

where the first term is due to thermal collisional excitations, the second to recombinations, and the third to direct non-thermal excitations by the fast non-thermal electrons from the $\gamma$-ray thermalization. The luminosity due to a collisionally excited line with excitation temperature $T_{e x}=h c / k \lambda$ decreases exponentially with falling temperature, to a first approximation $L_{i j} \propto T_{e}^{-0.5} e^{-T_{e x} / T_{e}}$. The recombination emission is less sensitive to temperature, and actually increases slowly, $L_{i j} \propto T_{e}^{-0.7}$, typically. Finally, the nonthermal excitation rate is independent of $T_{e}$. As discussed above, optical and near-IR lines have high excitation temperatures $T_{e x} \gtrsim 1 \times 10^{4} \mathrm{~K}$, and their luminosity decreases rapidly with temperature for $T_{e} \lesssim 3000 \mathrm{~K}$. The far-IR lines are much less sensitive 


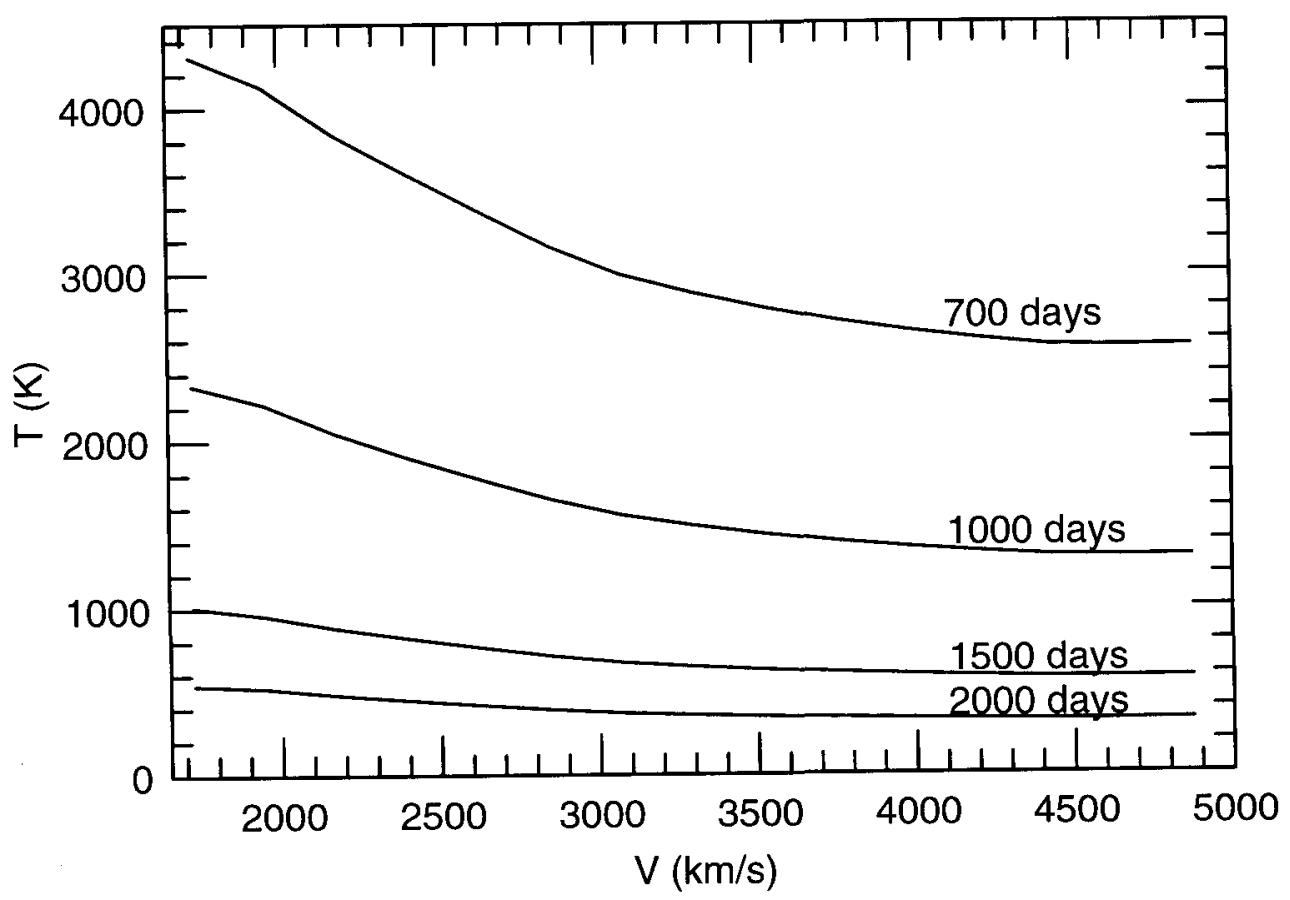

Figure 2. Temperature in the hydrogen envelope for different times.

with $T_{e x} \lesssim 1 \times 10^{3} \mathrm{~K}$. Therefore, as the temperature in the core drops with the IRcatastrophe, the collisional excitation contribution of the optical and near-IR lines drops dramatically. Lines which have no recombination contribution, like [O I] $\lambda \lambda 6300-64$, $\mathrm{Mg} \mathrm{I}] \lambda 4571$, [Ca II] $\lambda 7300$, and [Si I] $\lambda \lambda 1.099,1.607-1.645 \mu$ should display this behavior. This was predicted by the first models for the spectral evolution of SN 1987A (Fransson \& Chevalier 1987), and was later basically verified by the observations (e.g. Menzies 1991; Spyromilio et al. 1991). The decrease in the [Si I] line has been interpreted as a result of dust formation (Lucy et al. 1991), but our calculations show that this need not be the case, and that the amount of dust is therefore not constrained by these observations.

Observations of the emission lines from SN 1987A show a dramatic shift in their evolution at $\sim 700$ days. This is seen most clearly when the luminosities of the lines relative to the bolometric luminosity, $L_{j} / L_{b o l}$, are plotted against time (e.g. Menzies 1991). This behavior is exactly what is expected because of the IR-catastrophe. As the temperature drops from $\sim 3000 \mathrm{~K}$ to a few $\times 10^{2} \mathrm{~K}$ because of the instability, the thermal contribution to the collisionally excited optical lines decreases to a negligible level. However, there is still a non-thermal contribution due to excitation by the fast electrons which is independent of the temperature, as shown by the third term in equation (2.8). The luminosity of a line $j$ relative to the total bolometric luminosity in this phase is then

$$
\frac{L_{j}}{L_{b o l}}=\frac{\int \epsilon_{i, j} J_{\gamma} \sigma_{\gamma, i} n_{i} d V}{\int J_{\gamma} \sigma_{\gamma} n d V}=\epsilon_{i, j} \frac{\tau_{\gamma, i}}{\tau_{\gamma, t o t}}
$$

where $\tau_{\gamma, i}$ is the optical depth of the $\gamma$-rays to the ion $\mathrm{i}$ and $\tau_{\gamma, t o t}$ is the total $\gamma$-ray optical depth averaged over the ejecta according to the $\gamma$-ray intensity. Since both $\tau_{\gamma, i}$ 
and $\tau_{\gamma, t o t}$ vary with time as $t^{-2}$, we expect a fairly constant ratio of $L_{j} / L_{b o l}$. Actually, $\epsilon_{i, j}$ increases slowly with decreasing electron fraction, so a slight deviation from a constant is expected, as is indeed observed. From the bolometric light curve we find that $\tau_{\gamma, \text { tot }} \approx 0.5$ on day 1000. The $\epsilon_{i, j}$ 's are calculated from the Spencer-Fano equation (KF92) and we can therefore calculate $\tau_{\gamma, i}$ from $L_{j} / L_{b o l}$. At 1000 days we find from Menzies (1991) and Danziger et al. (1991) that for the [O I] $\lambda \lambda 6300-64$ lines $L_{6300} \approx 3.5 \times 10^{35}$ ergs, or $L_{6300} / L_{b a l} \approx 2.9 \times 10^{-3}$ (see figure 3 ). For a typical value of $x_{e}=10^{-2}$ at 1000 days $\epsilon_{6300} \approx 5 \times 10^{-3}$. The optical depth at 1000 days to the $\gamma$-rays in the oxygen gas is therefore $\tau_{\gamma, O} \approx 0.25$. The line width is $\sim 1500 \mathrm{~km} \mathrm{~s}^{-1}$. Including $\gamma$-ray input from $0.07 \mathrm{M}_{\odot}{ }^{56} \mathrm{Ni}$ and $2.5 \times 10^{-3} \mathrm{M}_{\odot}$ of ${ }^{57} \mathrm{Ni}$ (1.5 times solar) we estimate the [O I] $\lambda \lambda 6300-64$ luminosity from

$$
\begin{aligned}
L_{6300}= & 1.24 \times 10^{38} D_{\gamma}\left(e^{-t / 111.3}+9.3 \times 10^{-4} e^{-t / 391.2}\right) \times \\
& \left(\frac{V}{1500 \mathrm{~km} \mathrm{~s}^{-1}}\right)^{-2}\left(\frac{t}{1000 \mathrm{~d}}\right)^{-2}\left(\frac{M_{O}}{1 \mathrm{M}_{\odot}}\right) \text { ergs } .
\end{aligned}
$$

Unfortunately, the oxygen mass is fairly sensitive to the exact velocity distribution of the oxygen, as well as the nickel distribution, through $D_{\gamma}$ (KF92). However, generally $D_{\gamma} \lesssim 3$, and therefore $M_{O} \gtrsim 3\left(V / 1500 \mathrm{~km} \mathrm{~s}^{-1}\right)^{2} \mathrm{M}_{\odot}$. To pin down the distributions of the nickel and oxygen, the line profiles are of great value, since they in principle contain this information. We are currently studying this issue. However, it is encouraging that even this simple type of analysis gives the correct order of the mass. The great advantage of this method of determining the mass is that it is independent of the temperature and ionization, in contrast to the earlier epochs when the mass depends exponentially on the uncertain temperature. It is also insensitive to clumping, since only the column density enters. Similar arguments can be used to determine the mass of the other abundant elements. Since the relative flux is directly proportional to the mass of the element, only fairly abundant elements produce lines with a non-thermal, constant contribution. Other than [O I] $\lambda \lambda 6300-64$, the most interesting are $\mathrm{MgI}] \lambda 4571$, [Si I] $\lambda 1.645 \mu$. All show a nearly constant $L_{j} / L_{b o l}$ after $\sim 700$ days (Menzies 1991; Meikle et al. 1993; Danziger et al. 1991; Suntzeff et al. 1991).

In figure 3 we show the [O I] $\lambda \lambda 6300-64$ luminosity from a more realistic calculation taking all atomic processes and elements in the oxygen zone into account. After oxygen the most abundant elements are silicon and sulphur. Other zones have not been included in this calculation since they contribute little to the total [O I] $\lambda \lambda 6300-64$ emission. We assume the oxygen blobs are distributed uniformly in the core with a filling factor adjusted to give an oxygen density in agreement with that determined by Spyromilio \& Pinto (1991) and Li \& McCray (1992). Two sets of calculations are done. In one the total mass of the oxygen zone was $1 \mathrm{M}_{\odot}$ while in the other it was $2 \mathrm{M}_{\odot}$, corresponding to pure oxygen masses of $0.8 \mathrm{M}_{\odot}$ and $1.6 \mathrm{M}_{\odot}$, respectively. We have also varied the expansion velocity between 1000 and $2000 \mathrm{~km} \mathrm{~s}^{-1}$, which probably covers most of the observed range. The corresponding filling factors are in the range $0.05-0.78$. The temperature evolution is shown for the O-zone in figure 1, with a thermal instability at $\sim 900$ days. We also show the observed [O I] $\lambda \lambda 6300-64$ luminosity taken from Danziger et al. (1991) as filled dots in figure 3. First we note the very good agreement in the shape of the light curves and the observed evolution, with a characteristic break at $\sim 750$ days. This is a direct consequence of the thermal evolution, and therefore confirms the occurrence of the IR-catastrophe. The level of the emission is sensitive to the expansion velocity and the total oxygen mass, as expected from equation (2.10). We find that for core velocities higher than $\sim 1000 \mathrm{~km} \mathrm{~s}^{-1}$, as are indicated from the line 


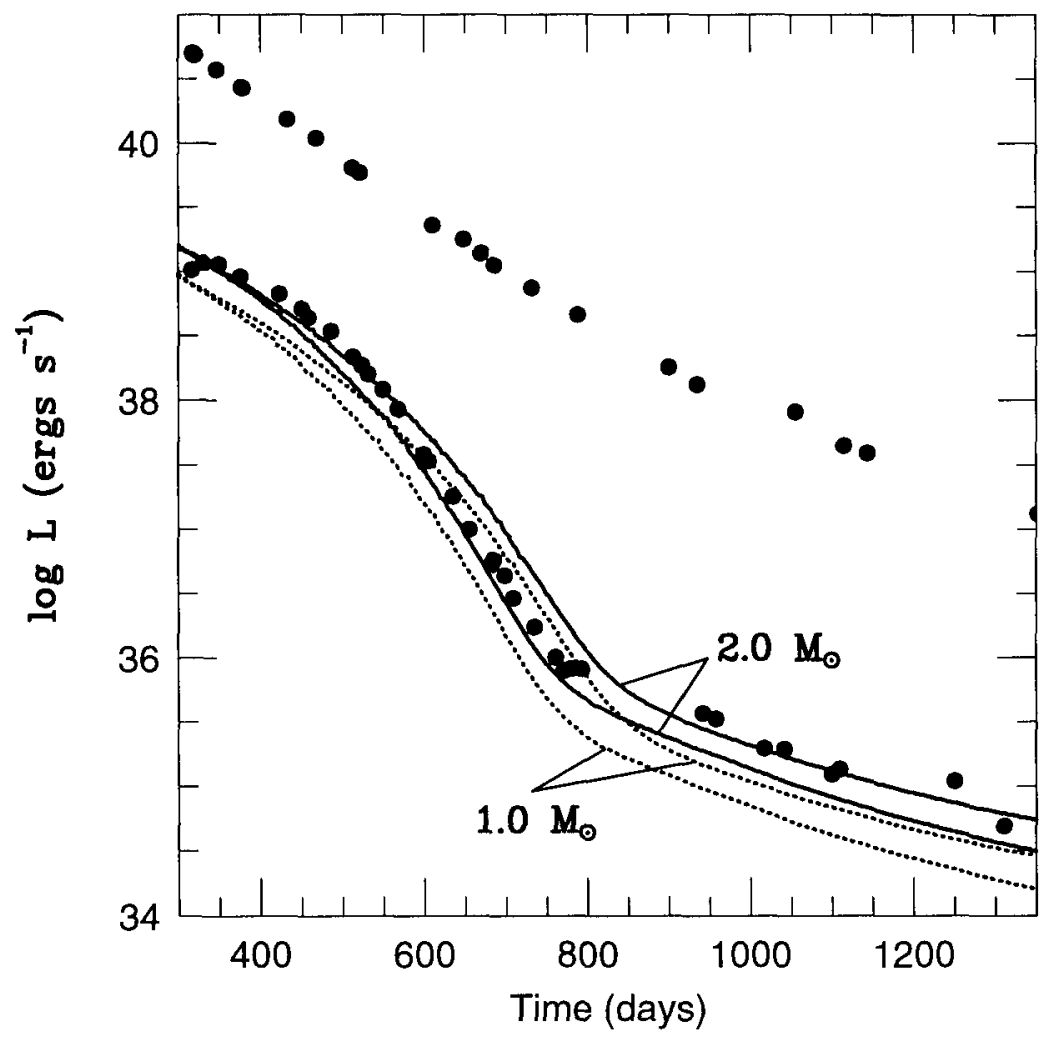

FIGURE 3. Luminosity of [OI] $\lambda \lambda 6300-64$ as a function of time, together with observations from Danziger et al. (1991). The lower curve for each mass corresponds to an expansion velocity of $2000 \mathrm{~km} \mathrm{~s}^{-1}$ and the upper to $1000 \mathrm{~km} \mathrm{~s}^{-1}$. The bolometric luminosity from Suntzeff et al. (1991) is shown as the upper dots. Before 750 days the emission is dominated by thermal excitations, while after this epoch non-thermal excitations dominate. This results in a nearly constant ratio of the line luminosity to the bolometric, as is observed.

profiles (e.g. Phillips \& Williams 1991), the oxygen mass must be at least $1.5 \mathrm{M}_{\odot}$. A smaller mass would not absorb enough $\gamma$-ray energy because of its small optical depth. Lower masses underestimate both the thermal and non-thermal parts of the light curve. The fact that we can fit both the flat, non-thermal part of the light curve after $\sim 750$ days and the thermal part before this epoch also means that the calculated temperature evolution is close to the actual temperature evolution.

The strongest line during most of the evolution is $\mathrm{H} \alpha$. The emission of this line has for SN 1987A been discussed by $\mathrm{Xu}$ et al. (1992) and in KF92. H $\alpha$ is formed mainly by recombinations. Xu et al. find that during the first $\sim 500$ days the non-thermal ionizations and excitations from the ground state are not enough to explain the line, but proposed that photoionization from $n=2$ by the Balmer continuum can account for the extra ionizations needed. This mechanism was discussed earlier by Kirshner \& Kwan (1974). KF92 analyzed the diffuse emission from the helium and metal core in order to calculate the UV radiation field and thus the strength of $\mathrm{H} \alpha$. The emission spectrum from the core is formed by a step-wise degradation of far-UV recombination lines, resulting from $\gamma$-ionizations in the metal core, into lines in the optical and Balmer continuum. An example is $\mathrm{He} I$ for which $\sim 20 \%$ of the recombination energy emerges 
directly as optical lines. The rest is emitted mainly as two-photon emission between $0-$ $20.6 \mathrm{eV}$. In the He-zone, $\mathrm{C}$ is the second most abundant element, making the gas opaque above $11.26 \mathrm{eV}$. The two-photon emission between $11.26 \mathrm{eV}$ and $20.6 \mathrm{eV}$ therefore results in ionizations of $\mathrm{C} I$, followed by C I recombination emission. Most of this is emitted as UV lines around $\sim 1200 \AA$, which can then be absorbed by the Balmer continuum. In the iron core, where helium has an abundance of $\sim 0.56$ by number, Fe I plays the same role as C I, but with an ionization threshold of $7.87 \mathrm{eV}$. This example shows that a fairly complicated transfer of energy takes place from one zone (or element) to another, and that large errors will be introduced if these effects are not taken into account. KF92 find that a fraction $\epsilon_{U V} \approx 30 \%$ of the total energy absorbed by the He- and metal core is emitted in the Balmer continuum. In the $\mathrm{H}$-rich gas the recombinations and excitations ending up in the $n=2$ level of $\mathrm{H}$ I will decay by two-photon emission. A fraction $\sim 0.88$ of this is above $3.4 \mathrm{eV}$ and adds to the Balmer ionization. At $x_{e} \approx 10^{-2}$, $\chi_{\text {eff }} \approx 34 \mathrm{eV}$, and a fraction $\chi_{i} / \chi_{\text {eff }} \approx 40 \%$ of the $\gamma$-ray energy absorbed directly in the hydrogen gas goes into ionizations, and $32 \%$ into excitations. The total energy of the two-photon emission above $3.4 \mathrm{eV}$ is then $\sim 0.88 \times(0.75 \times 0.40+0.32) \tau_{H} L_{\gamma}=0.56 \tau_{H} L_{\gamma}$. Therefore, the ratio of Balmer ionizations to those from the ground state is

$$
\frac{\zeta_{\text {Balmer }}}{\zeta g . s .}=\frac{\left(\epsilon_{U V} \tau_{\text {core }}+0.56 \tau_{H}\right)}{\tau_{H}} \frac{\chi_{e f f}}{\chi_{n=2}} \approx\left(3.0 \frac{\tau_{\text {core }}}{\tau_{H}}+5.6\right) .
$$

Below, we find that $\tau_{\text {total }} / \tau_{H} \approx 2.5$, so $\tau_{\text {core }} / \tau_{H} \approx \tau_{\text {total }} / \tau_{H}-1 \approx 1.5$ and $\zeta_{\text {Balmer }} / \zeta g . s . \approx$ 10 , which is larger than the factor $\sim 5$ needed by Xu et al. (1992).

The fraction of the luminosity emitted as $\mathrm{H} \alpha$ shows a marked decrease at $\sim 700$ days, after which it remains approximately constant (e.g. Menzies 1991). The decrease is not a temperature effect, but mainly a result of the Balmer continuum becoming optically thin. After $\sim 500$ days the excitation of the line is dominated by non-thermal ionizations, followed by recombinations, plus direct non-thermal excitations to the $n=3$ level. The expected decrease is therefore a factor $\sim 10$ from equation (2.11), in reasonable agreement with the observations. If we neglect freeze out effects for the moment, we find that

$$
\begin{aligned}
\frac{L_{\mathrm{H} \alpha}}{L_{b o l}} & =\left(\frac{\chi_{i}}{\chi_{e f f}} \frac{j_{\mathrm{H}_{\alpha}}}{\alpha_{B} \chi_{i}}+\frac{5}{36} \epsilon_{H, n=3}\right) \frac{\tau_{\gamma, H}}{\tau_{\gamma, t o t}} \\
& \approx(0.4 \times 0.070+0.14 \times 0.05) \frac{\tau_{\gamma, H}}{\tau_{\gamma, t o t}}=0.035 \frac{\tau_{\gamma, H}}{\tau_{\gamma, t o t}} .
\end{aligned}
$$

If the Balmer lines are optically thick (Case $\mathrm{C}$ ), the $\mathrm{H} \alpha$ strength may increase by a factor $\sim 2$ compared to this estimate. Freeze out effects are important after $\sim 900$ days in the $\mathrm{H}$ regions (FK93), and after this epoch the delayed recombination will give an additional contribution to $\mathrm{H} \alpha$. On day $750 L_{\mathrm{H}_{\alpha}} / L_{b o l} \approx 1.4 \times 10^{-2}$ and we find that $\tau_{\gamma, H} / \tau_{\gamma, \text { tot }} \approx 0.40$, or translated to 1000 days $\tau_{\gamma, H} \approx 0.20$. This value is somewhat larger than from the Shigeyama et al. (1988) 14E1 model, and may indicate an hydrogen mass less than $\sim 10 \mathrm{M}_{\odot}$.

Finally, we note that the evolution of the line luminosities in general offer an interesting possibility to test the radioactive decay time scale, and thus the dominant source of radioactivity or a possible pulsar contribution. Because lines fed by direct excitation decay immediately to the ground state, the luminosity will follow the instantaneous energy input,

$$
L_{j} \propto \epsilon_{i, j} \tau_{i} e^{-t / \tau} \propto \epsilon_{i, j} t^{-2} e^{-t / r}
$$

if $\tau_{\gamma}<<1$. This applies to e.g. the [OI] line. If positrons from ${ }^{44} \mathrm{Ti}$ dominate the energy input $L_{j} \propto \epsilon_{i, j} e^{-t / \tau} \approx$ constant. A line mainly arising due to recombination will behave 
like

$$
L_{j} \propto \int j\left(T_{e}\right) n_{e} n_{i+1} d V \propto j\left(T_{e}\right) x_{e}^{2} t^{-3} \propto x_{e}^{2} t^{-1.6}
$$

in the freeze out phase, since for H-like ions $j\left(T_{e}\right) \propto T_{e}^{-0.7} \propto t^{1.4}$, if adiabatic cooling dominates.

\section{Type Ia SNe}

While considerable progress has been made on the late emission from Type II SNe, the situation is worse for Type Ia SNe both observationally and theoretically. Few detailed observations exist. In fact, only for SN 1972E does there exist good photometry and spectra beyond a year (Kirshner et al. 1973, 1975). Our ability to produce detailed synthetic spectra is also rather limited, in part because of the continuing lack of high quality atomic data for iron ions.

Even neglecting the absence of hydrogen, the physical conditions in Type Ia SNe are quite different from Type II's. In the best studied model, in which a Type Ia supernova is produced by the explosion of a Chandrasekhar mass white dwarf, the total mass $\lesssim 1.4 \mathrm{M}_{\odot}$ is smaller than that of a massive stellar core by a factor $\sim 3$. The observed expansion velocity of this metal rich material $\left(\sim 10^{4} \mathrm{~km} \mathrm{~s}^{-1}\right)$ is considerably greater than the $\sim 2000 \mathrm{~km} \mathrm{~s}^{-1}$ typical in the Type II's, so that the total density is smaller by at least a factor $\sim 400$ in the Type Ia case. Given that the ${ }^{56} \mathrm{Ni}$ mass produced in a Type Ia supernova is also larger by a factor $\sim 10$ the level of ionization is much higher than in a Type II at a similar epoch (e.g. equation 1.3).

To study the spectral evolution and the photometric light curves, Houck, Fransson \& Pinto (1994) calculated the time dependent spectrum for several different explosion models taken from Woosley (1991), Woosley (1992, private communication) and Woosley et al. (1986). A time dependent calculation is necessary to treat the freeze out effects properly. Unfortunately, the exact results depend fairly sensitively on the recombination rates of $\mathrm{Fe} \mathrm{I}-\mathrm{Fe} \mathrm{V}$, which are uncertain by embarrassingly large factors. Hopefully, new calculations within the OPACITY project can improve this situation (Le Dourneuf et al. 1993). Using rates by Shull \& Van Steenberg (1982) we find that the level of ionization at 250 days is much too high to fit the observed optical spectrum of SN 1972E. Because increasing the Fe II and Fe III recombination rates by a factor $\sim 3$ considerably improves the agreement with the observed spectrum, we calculate the light curves using these enhanced recombination rates instead of the Shull \& Van Steenberg rates. This is the primary uncertainty in the model.

Here we describe results for model DD4 from Woosley (1991), corresponding to a "delayed detonation" of a Chandrasekhar mass white dwarf producing $0.62 \mathrm{M}_{\odot}$ of mainly ${ }^{56} \mathrm{Ni}$. This model produces the best fit to the observed optical spectrum of SN 1972E at 250 days. In figure 4 we show the temperature and ionization evolution for a number of different mass elements from the center to the surface. First, we note that the most abundant ion is Fe II even at very late stages, leading to $x_{e} z 0.5$ as late as $\sim 900$ days. This confirms the high level of ionization expected. The low ionization zone corresponds to the central region where the ${ }^{56} \mathrm{Ni}$ concentration is quite low. The temperature curves clearly show the onset of the thermal instability at $\sim 450$ days; over the next 200 days, the temperature rapidly decreases from $\sim 3000 \mathrm{~K}$ to $\sim 300 \mathrm{~K}$. This is the IR-catastrophe originally discussed by Axelrod (1980). After the IR-catastrophe nearly all emission emerges as fine structure lines of [Fe II] $(\lambda 25.99 \mu, \lambda 35.35 \mu)$ and [FeI] $(\lambda 24.05 \mu)$.

From the calculated spectra we determine the flux in various photometric bands, and 


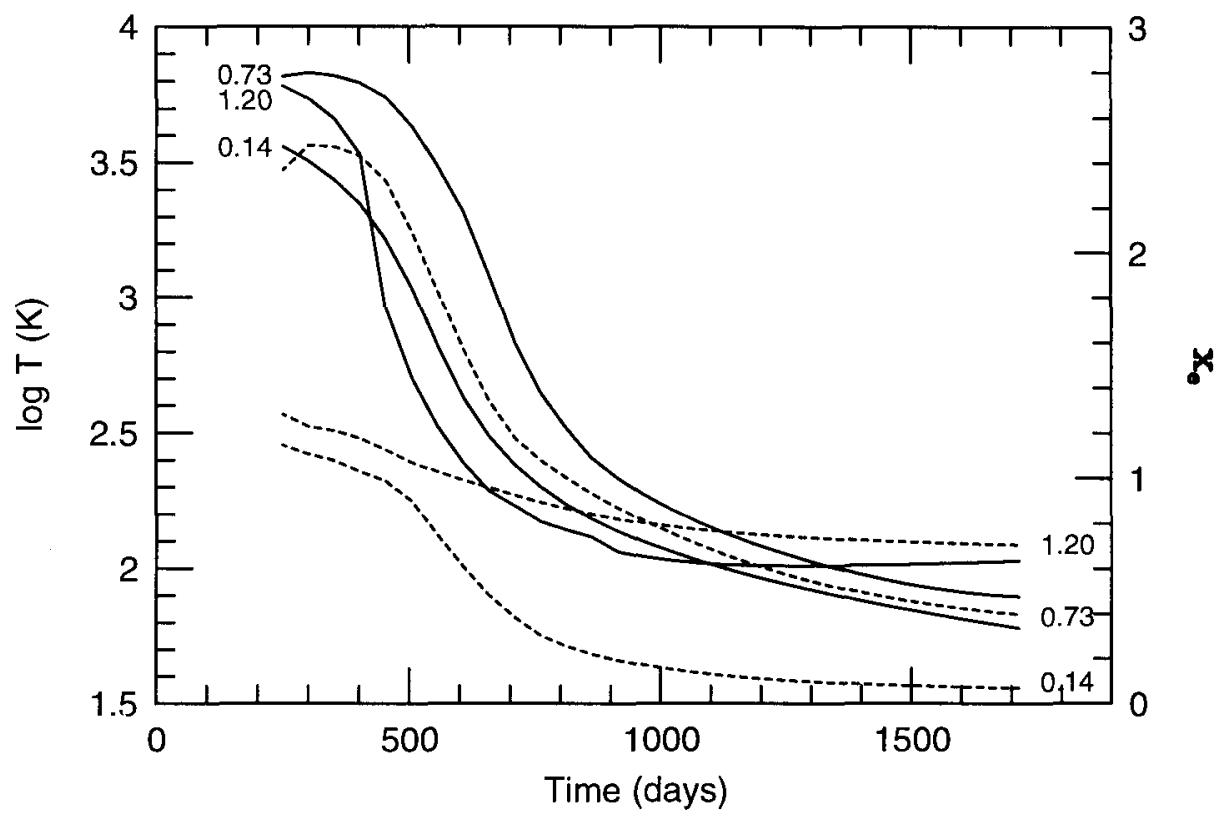

Figure 4. Time evolution of the temperature (full lines) and electron fraction (dashed lines) for three different mass shells in the Type Ia SN model DD4 from Woosley (1991). Mass shells with interior mass $M\left(R_{i}\right)=0.14,0.73$, and $1.20 \mathrm{M}_{\odot}$ are shown. Note the thermal instability at 500 - 800 days, and the high degree of ionization even beyond 1000 days.

in figure 5 we show the resulting light curves from the UV to the IR. We have here used a distance of $4 \mathrm{Mpc}$, appropriate for SN 1972E. For a more common distance of $10 \mathrm{Mpc}$ one should add 2 magnitudes. There is a marked decrease in the fluxes in most of the optical bands between 450 and 600 days, coinciding with the IR-catastrophe. The $\mathrm{V}$ magnitude increases from $\mathrm{V} \approx 18.2$ to $\mathrm{V} \approx 22.8$, while the $\mathrm{B}$ magnitude increases from $\mathrm{B} \approx 18.3$ to $\mathrm{B} \approx 23.2$. The $\mathrm{U}$ band, however increases more smoothly out to 500 days. The $\mathrm{J}$ magnitude appears to rise quite slowly with $\mathrm{J} \approx 16$ from 300 until 500 days, when it also increases rapidly $\mathrm{J} \approx 24.4$ at 700 days. The slow decline in $\mathrm{J}$ happens at the same time as the rapid decline in $\mathrm{B}$ and can be attributed to a shift in emission from the strong $[\mathrm{Fe}$ III] blend at $\sim 5000 \AA$ to the [Fe II] lines at $1.257 \mu$ and $1.644 \mu$ in the near-IR. Despite the rapid cooling during this epoch, we find no brightening in the far-IR photometry because the strongest fine structure lines, e.g. [Fe II] $\lambda 25.99 \mu, \lambda 35.35 \mu,[\mathrm{Fe} I] 24.05 \mu$ are outside the photometric bands, and no strong lines lie in the $\mathrm{N}$ and $\mathrm{Q}$ bands. Possibly ISO may be sensitive enough to measure the fluxes in the interesting bands (especially the $20-36 \mu$ region).

The rapid increase in $B$ magnitude between 450 and 700 days is inconsistent with the $B$ light curve of SN 1972E obtained by Kirshner et al. (1975). However, the proper means for avoiding the rapid drop in the computed optical luminosity compared to the near IR is not clear. As in the Type II case, this emission is mainly due to recombinations following non-thermal ionizations which, as was discussed earlier, are relatively insensitive to the temperature. In the Type Ia case, because the electron fraction is $x_{e} Z 0.5$ until very late times, $t \sim 900$ days, most of the non-thermal energy deposited during this epoch goes 


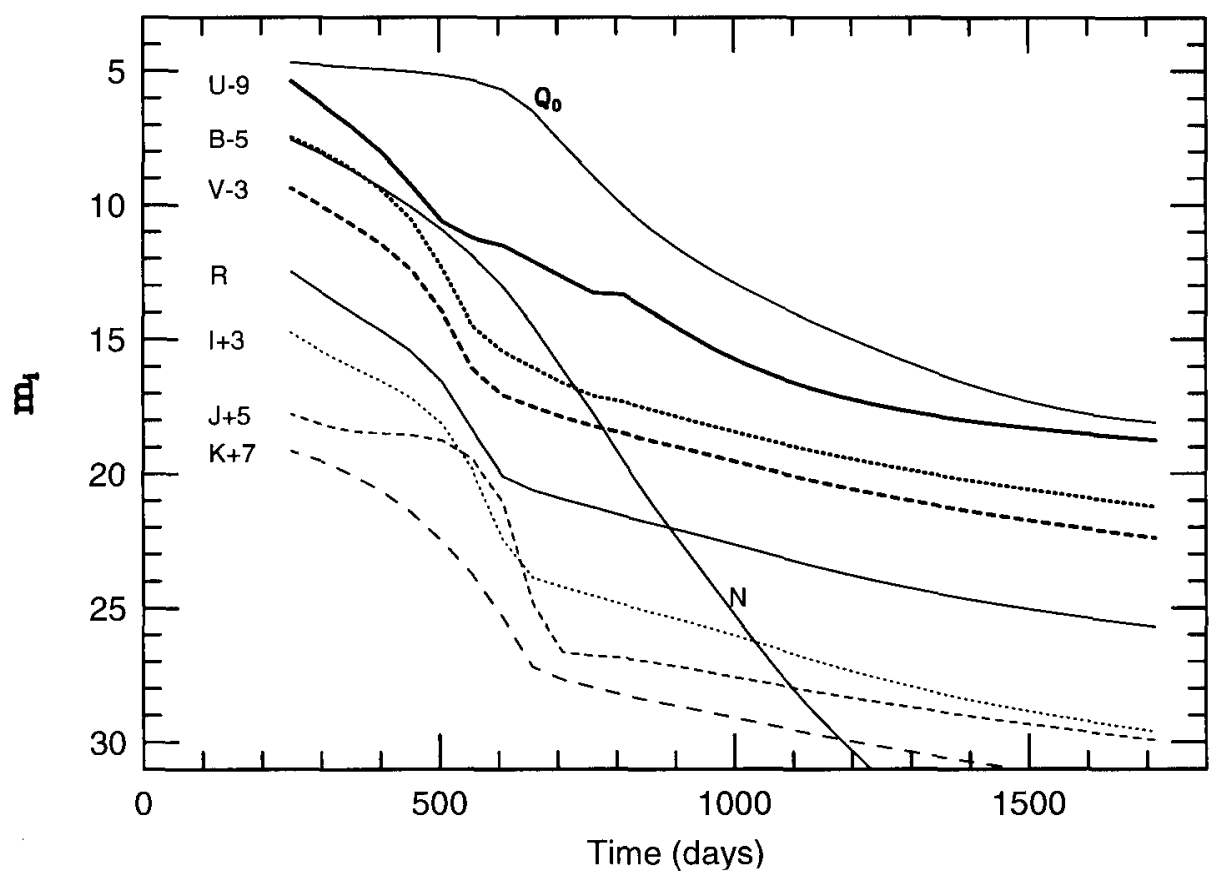

Figure 5. Photometric light curves for the same model as in figure 4, for a SN distance of 4 Mpc. The decrease at $\sim 600$ days is due to the thermal instability.

into heating and ionization and only a negligible fraction goes into direct excitation. Our computed spectrum at 400 days gives a reasonably good fit to the $[\mathrm{Fe} I \mathrm{II}] \lambda 1.257 \mu$ and [Fe II] $\lambda 1.644 \mu$ features observed in SN 1991T by Spyromilio et al. (1992), however the optical features in our computed spectrum are too faint by factors $\sim 3-5$. One possible explanation is that our model temperature is too low, perhaps because of clumping in the ejecta. In this case, most of the optical emission might come primarily from the hotter lower density regions between the clumps. Another possibility is that the optical emission, which should come from the recombination cascade, is modeled incorrectly because of the lack of accurate atomic data.

Because of its larger region of higher ${ }^{56} \mathrm{Ni}$ concentration, model DD3 $\left({ }^{56} \mathrm{Ni}\right.$ mass $0.93 \mathrm{M}_{\odot}$ ) from Woosley (1991) produces a more gradual decrease in the $\mathrm{B}$ light curve, with the $\mathrm{B}$ magnitude falling to $\mathrm{B} \approx 23$ only after $\sim 700$ days. The higher ${ }^{56} \mathrm{Ni}$ concentration produces a higher level of ionization and heating so that the IR-catastrophe is postponed. However, even this more extreme model recombines and cools to rapidly to fit the observed light curve of SN 1972E.

At epochs later than $\sim 1000$ days the light curves stay fairly constant as a result of both the extra energy input from ${ }^{44} \mathrm{Ti}$ and a freeze out. Which of these is the most important is sensitive to the mass of ${ }^{44} \mathrm{Ti}$, which in turn depends on the particular explosion model. Unfortunately, it will not be easy to distinguish these two effects. Because of recombination to $\mathrm{Fe} \mathrm{I}$ at very late times $t>1500$ days, the supernova brightens in the optical so that as much as $50 \%$ of the luminosity comes out shortward of $1 \mu$. However, the details of this result depend on the low temperature $(\sim 100 \mathrm{~K}) \mathrm{Fe}$ I recombination rate and are quite uncertain.

In conclusion, although spectroscopy later than a year is not trivial, photometry should 
be fairly easy, and may therefore be the best way to study these phases. Photometry is also likely to be less sensitive to uncertainties in the atomic data. Even in this case, the quality of the atomic data for $\mathrm{Fe}$ is the main obstacle in modeling Type Ia spectra.

\section{REFERENCES}

Axelrod, T.S., (1980) Ph.D. thesis, Univ. of California, Santa Cruz.

Chevalier, R. A., \& Fransson, C., (1992) ApJ, 395, 540

Danziger, I.J., Gouiffes, C., Bouchet, \& P. Lucy L.B. , (1991), ESO/EIPC Workshop Supernova 1987 A and Other Supernovae, eds. I.J. Danziger \& K. Kjär, p. 217

Le Dourneuf, M., Nahar, S.N., \& Pradhan, A.K., (1993) J. Phys. B, 26, L1

Fransson, C., (1993) in Les Houches, Session LIV, 1990, Supernovae eds. J. Audouze, S. Bludman, R. Mochkovitch and J. Zinn-Justin, Elsevier Science Publishers B.V., in press

Fransson, C., \& Chevalier, R.A. , (1987) ApJ, 322, L15

Fransson, C., \& Chevalier, R. A., (1989) ApJ, 343, 323

Fransson, C., \& Kozma, C., (1993) ApJ, 408, L25 (FK93)

Fryxell, B., Müller, E. \& Arnett, D., (1991) ApJ, 367, 619

Houck, J., Fransson, C., \& Pinto P., (1993) in preparation

Kirshner, R.P., \& Kwan, J. , (1975) ApJ, 197, 415

Kirshner, R.P., Oke, J.B., Penston, M.V., \& Searle, L., (1973), ApJ, 185, 303

Kirshner, R.P., \& Oke, (1975), ApJ, 200, 574

Kozma, C., \& Fransson, C., (1992), ApJ, 390, 602 (KF92)

Kurfess, J.D. et al. (1992) ApJ, 399, L137

Li, H. \& McCray, R., (1992), ApJ 387, 309

Lucy, L.B., Danziger, I.J., Gouiffes, C., \& Bouchet P. , (1991) Supernovae, Proc. of the Tenth Santa Cruz Summer Workshop in Astronomy and Astrophysics, ed. S.E. Woosley, Springer Verlag, 82

Menzies, J.W. , (1991), ESO/EIPC Workshop Supernova 1987A and Other Supernovae, eds. I.J. Danziger \& K. Kjär, p. 209

Meikle, W.P.S., Spyromilio, J., Allen, D.A., Varani, G.-F., \& Cumming, R.J., (1993) MN 261, 535

Phillips, M.M., \& Williams, R.E. (1991) Supernovae, Proc. of the Tenth Santa Cruz Summer Workshop in Astronomy and Astrophysics, ed. S.E. Woosley, Springer Verlag, 36

Pinto, P.A. \& Woosley, S.E., (1988) Nature, 333, 534

Shigeyama, T., Nomoto, K., \& Hashimoto, M., (1988) A\&A, 196, 141

Shull, J.M., \& Van Steenberg, M., (1982), ApJS., 48, 95

Spyromilio, J. \& Pinto, P. A. (1991), ESO/EIPC Workshop Supernova 1987 A and Other Supernovae, eds. I.J. Danziger \& K. Kjär, p. 423

Spyromilio, J., Stathakis, R.A., Cannon, R.D., Waterman, L. \& Couch, W.J., (1991) MN 248, 465

Suntzeff, N.B., Philips, M.M., Depoy, D.L., Elias, J.H., \& Walker, A.R., (1991) AJ, 102, 1118

Woosley, S.E., (1991), Gamma-Ray Line Astrophysics, eds. P.Durouchoux \& N. Prantzos, (New York: American Institute of Physics), 270.

Woosley, S. E., Pinto, P. A., \& Hartmann, D., (1989) ApJ 346, 395

Woosley, S.E., Taam, R.E., \& Weaver, T.A., (1986), ApJ 301, 601

Xu, Y., \& McCray, R. (1991) ApJ, 375, 190

Xu, Y., McCray, R., Oliva, E., \& Randich, S. (1992) ApJ, 386, 181 